\title{
EDITORIAL
}

\section{Una perspectiva de la educación en enfermería: investigación para el desarrollo de la disciplina}

\section{A perspective of nursing education: research for discipline development}

\begin{abstract}
a formación en enfermería y su preponderante necesidad de progresar debe considerar la evolución tecnológica, educativa y social, así como la modificación en el perfil epidemiológico de la población. Todo esto requiere recursos humanos capacitados - para responder a la diversidad de circunstancias, para comprender los factores que influyen en el continuum saludenfermedad de la población (1). Lo anterior, asociado a la situación que afecta a la educación superior, que incita a las escuelas de enfermería a evolucionar, reflexionando acerca de la misión de generar enseñanza de calidad, para responder a las necesidades que presentan la población, los servicios de salud y de la comunidad. Todo esto permite que se desarrollen mecanismos que vinculen apropiadamente educación, atención en salud e investigación en enfermería.
\end{abstract}

La educación e investigación en enfermería cimientan el desarrollo y progreso de la profesión. Su crecimiento, modernización e innovación, exigen cambios que respondan al avance, mejora y perfeccionamiento de programas de formación, tanto de pregrado y posgrado, como en aquellos dirigidos al equipo de salud o a los usuarios. Además, la investigación como función de enfermería, le permite argumentar su conocimiento y habilidades en relación con su objeto de estudio, el cuidado, así como llevar a cabo estudios científicos necesarios que aporten nuevos paradigmas a la disciplina.

La investigación como método progresivo busca nuevos objetivos de conocimiento en la creación y reforzamiento de las actividades encaminadas al desarrollo de la profesión, relacionados con el hacer y cuestionan el saber de Enfermería. La investigación disciplinar respalda un conjunto de saberes y responsabilidades frente a los retos que plantea la globalización y sus efectos en la salud de la sociedad mexicana.

La investigación en enfermería fundamenta la práctica clínica y el pensamiento crítico, guiándonos hacia la autonomía profesional. Para hacer frente a los determinantes de salud actuales, se debe considerar que los profesionales de Enfermería cumplen con su compromiso ético y legal ante la sociedad al prepararse continuamente para dicho cometido.

Por último, la educación superior en el Departamento de Enfermería de la Universidad de Sonora ha evolucionado a través de la planificación e implementación de procesos de autoevaluación y evaluaciones externas adecuados. La calidad en la educación exige como prioridad planificar y efectuar mediciones de calidad, renovadas y congruentes, que den cuenta de las fortalezas y debilidades de las acciones educativas, para realizar las modificaciones y/o correcciones necesarias, como la de reflexionar de manera colegiada acerca del camino que se tiene que recorrer como profesionales comprometidos para argumentar y evaluar la importancia de la investigación en la disciplina, lo que fortalecerá su autonomía e identidad profesional.

La investigación constituye junto con la gestión, la práctica y la educación, uno de los 4 pilares en los que se cimienta la disciplina de Enfermería.

\section{Dra. Rosa María Tinajero González \\ Doctora en Educación \\ Universidad de Sonora \\ Maestra de tiempo completo \\ Departamento de Enfermería}

rosa.tinajero@unison.mx

\section{REFERENCIAS BIBLIOGRÁFICAS}

1. Miotto, M. da G.; Garzon, N. Estudio de los programas de especialización y maestría en enfermería en América Latina. Educ.Med.Salud. 1995: 29 (3-4); 331-43. 